\title{
A MEMS-Controllable Fresnel Zone Plate for Miniaturized UV Spectrometer ${ }^{\dagger}$
}

\author{
Elnaz Afsharipour *, Pawel Glowacki and Cyrus Shafai \\ Department of Electrical and Computer Engineering, University of Manitoba, Winnipeg, MB R3T 2N2, \\ Canada; pawel.k.glowacki@gmail.com (P.G.); Shafai@umanitoba.ca (C.S.) \\ * Correspondence: afsharie@myumanitoba.ca; Tel.: +1-204-698-0661 \\ + Presented at the Eurosensors 2017 Conference, Paris, France, 3-6 September 2017.
}

Published: 5 September 2017

\begin{abstract}
This work presents the design and simulation of a MEMS-controllable linear motion Fresnel Zone Plate (FZP). The focal length of an FZP is a function of incident wavelength. This principle can be used for separating wavelengths, enabling a spectrometer application. The FZP of this work is designed on a linearly moving MEMS actuator, enabling wavelength focusing onto a fixed image plane. The FZP is designed with 400 zones, which can focus wavelengths from $150 \mathrm{~nm}$ to $410 \mathrm{~nm}$ by moving on a distance of $1 \mathrm{~mm}$.
\end{abstract}

Keywords: Fresnel Zone Plate; MEMS FZP; Lorentz force actuator; miniaturized spectrometer; diffractive wavelength selection

\section{Introduction}

MEMS actuators have been used as the platform of optical components including mirrors [1], gratings [2], and lenses [3] for beam steering, focusing and separating light wavelengths. For example, scanning mirrors fabricated on top of MEMS actuators have been successfully reported in OCT probes or in vivo catheters [3]. MEMS controlled lenses have also been fabricated to accurately align the focused light to a detector. Reference [2] fabricated a diffraction grating on top an actuator to separate the wavelengths of light which will be detected by a CCD. Fresnel Zone Plate (FZP) is another optical component, that has been used for selective focusing. FZPs have shown to be an effective diffractive element for miniaturized, transportable spectrometers designed for specific applications. For example, [4] employed multiple FZPs for specific wavelength selection. An FZP focuses the incident light at different distances based on its wavelength. Selecting which wavelength passes through the aperture is possible by moving the actuator over the focusing axis. Using this method miniaturized and transportable spectrometers using FZPs can be fabricated.

In this work, we present a MEMS to linearly adjust the FZP position, enabling analog control of FZP wavelength focusing. A simplified setup of the system is shown in Figure 1. The FZP is embedded on top of a $2 \mathrm{~mm} \times 2 \mathrm{~mm}$ membrane at the centre of a $10 \mathrm{~mm} \times 6 \mathrm{~mm}$ actuator. The rectangular membrane has 4 flexures at its corners and works based on Lorentz force. The serpentine flexures are designed to be flexible enough for motion while preventing deformation and curvature on the FZP plate. 


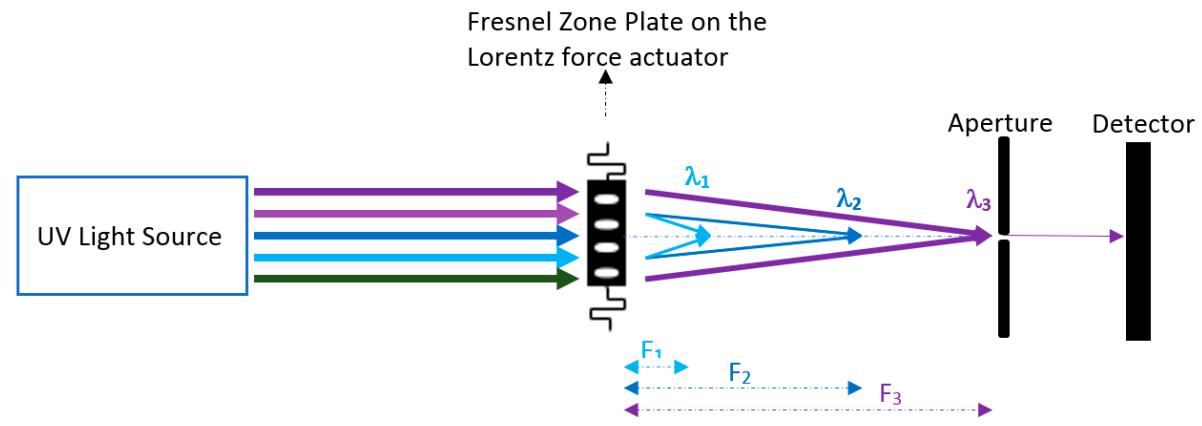

Figure 1. The FZP is placed on top of a MEMS actuator which moves horizontally. $F_{1}, F_{2}$, and $F_{3}$ are the focal lengths of $\lambda_{1}, \lambda_{2}$, and $\lambda_{3}$ respectively. By moving the FZP the desired wavelength can be focused on the detector.

\section{Materials and Methods}

Figure 2a shows the schematic of the structure. The design of the structure involves both mechanical and optical design. The mechanical design includes determining the actuator's physical parameters while in the optical design, factors affecting the focusing ability of the FZP is discussed.

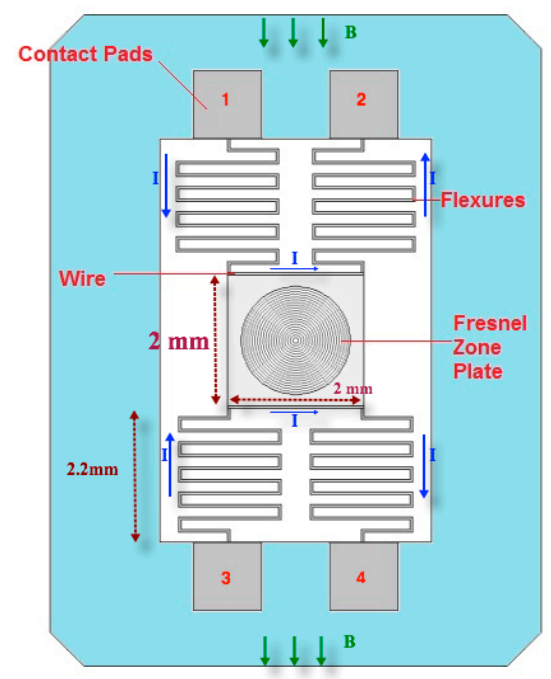

(a)

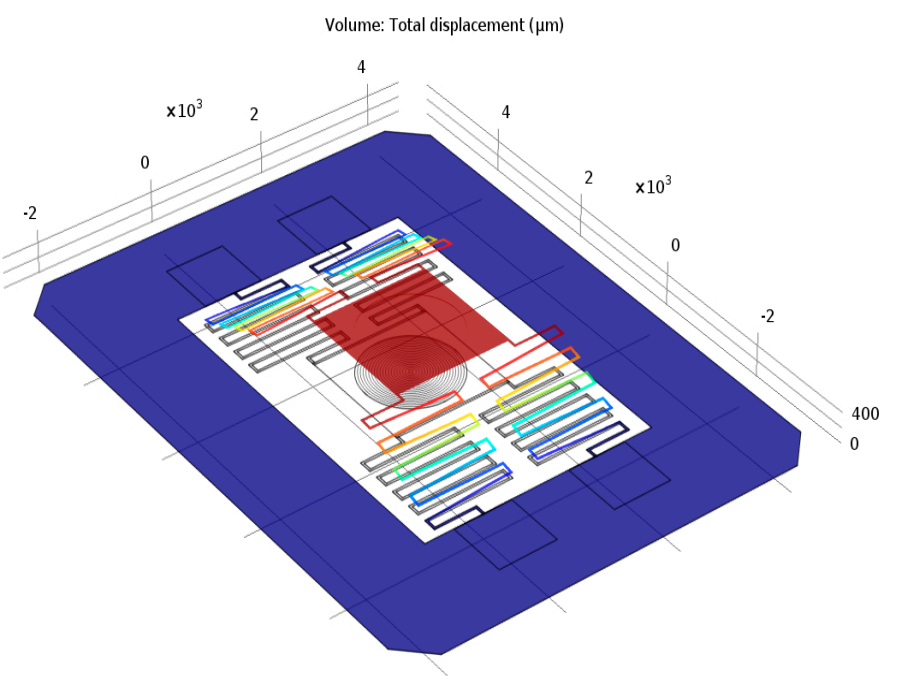

(b)

Figure 2. The schematic of the MEMS controlled FZP. The Fresnel zone plate is placed at the centre of the structure. (a) Front view of the structure; (b) 3D view of FZP in actuated mode.

\subsection{Mechanical Design}

As shown in Figure 2a, the actuator consists of a $2 \mathrm{~mm} \times 2 \mathrm{~mm}$ membrane upon which the FZP is fabricated. Four serpentine springs connect the membrane to the frame. An electric current $I$ is applied to the contact pads 1 and 3 . The current passes through the flexures and mirror sides $(L)$ and closes its loop on contact pads 2 and 4 . An external magnetic field $B$ is generated using a permanent magnet with a direction perpendicular to the mirror sides. According to the equation, $\overrightarrow{\mathrm{F}}_{\text {Lorentz }}=$ $\overrightarrow{\mathrm{I}} \mathrm{L} \times \overrightarrow{\mathrm{B}}$, a Lorentz force is exerted on the mirror sides. Since the direction of current in the effective length of flexures is parallel to the magnetic field, no force is applied on them. The membrane and substrate of wires are made of $5 \mu \mathrm{m}$ thick silicon. A layer of $1.5 \mu \mathrm{m}$ aluminum is used to make the conductive wires and the FZP opaque zones. The serpentine shaped spring wires are of $30 \mu \mathrm{m}$ width. The whole size of actuator is $10 \mathrm{~mm} \times 6 \mathrm{~mm}$. Figure $2 \mathrm{~b}$ shows the actuated mode of the structure. The membrane which is shown in red, has moved out of the plane. 


\subsection{FZP Design}

An FZP is made of concentric rings of opaque and transparent regions. When a wavefront of light hits the FZP, it gets diffracted by the opaque zones. Depending on the distance between two opaque zones, the light can make constructive interferences at a certain focal point. In the other words, at a particular focal point, all radiations of a certain wavelength are in phase that can constructively interfere, while most of the radiations of other wavelengths destructively interfere. In this work, the FZP is designed to focus the wavelength range of $150 \mathrm{~nm}$ to $410 \mathrm{~nm}$ in a focal distance of $1 \mathrm{~mm}$. Knowing the design wavelength and focal length, the physical parameters of FZP were determined. The physical parameters include:

1. The width of transparent rings.

2. The distance between the center of one transparent ring to the center of next transparent ring.

3. The distance between the first transparent ring and the center of the FZP plane.

4. Diameter of the aperture in the focal plane.

5. Number of transparent rings.

While calculating these parameters, the effectiveness of the FZP should also be considered. Therefore, the following parameters were used to evaluate the functionality of FZP at each step of calculating the physical parameters. These parameters affect the quality of constructive interference between photons at the specified focal point. The details of the algorithm can be find in [5].

1. The spatial FWHM (Full Width at Half Maximum) was designed to be narrow to increase the temporal coherency of the lens.

2. The transmission efficiency, or ratio of transmitted light through the lens, was maximized. This parameter is important to determine the minimum detectable photon energy.

3. The aperture restricted spectral radiosity, which is the ratio of spectral irradiance passing through the aperture, was maximized. This parameter is important for determining the diameter of the aperture.

4. The spectral resolution, which is the ratio of the central wavelength to the FWHM, was maximized. This parameter is important for getting a narrow bandwidth of desired wavelength at the output.

With these considerations, the FZP of this work is designed with 400 alternating opaque and transparent zones. The width of first transparent ring is $5 \mu \mathrm{m}$, and decreases to $245 \mathrm{~nm}$ for the last zone (400). The designed FZP will focus wavelengths ranging from $150 \mathrm{~nm}$ to $410 \mathrm{~nm}$, over the distance from $0.01 \mathrm{~mm}$ to $1 \mathrm{~mm}$ from the lens.

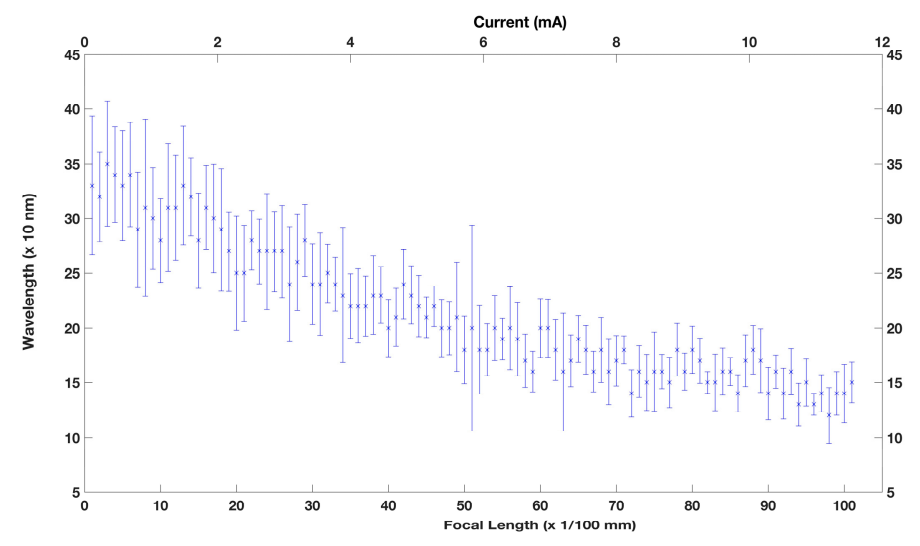

Figure 3. Focusing ability of the designed Fresnel zone plate. The horizontal axis shows the focal distance of the main harmonic of each wavelength. The MEMS actuator can move over a distance of $1 \mathrm{~mm}$, which can selectively focus UV wavelengths from $150 \mathrm{~nm}$ to $410 \mathrm{~nm}$ onto the detector. The top $x$ axis shows the amount of current that must be applied to the MEMS actuator of Figure 2, to displace the FZP. 


\section{Results}

The response function of the designed FZP is shown in Figure 3. The figure shows the wavelength with maximum intensity passing through the aperture, as a function of focal distance. At each point, the error bars show the standard deviation of distributed bandwidth of wavelengths that also pass through the aperture. The top $x$ axis shows the amount of current the MEMS actuator needs to displace the FZP.

\section{Conclusions}

In this work, a MEMS-controlled FZP is designed. The structure includes an FZP which is placed on top of a Lorentz force MEMS actuator. By applying the current to the actuator, the FZP linearly moves on its focal distance. Since the FZP is a diffractive-based lens, each wavelength of incident light is focused on a different focal point. Therefore, a desired wavelength can be focused on the aperture of the detector by moving the FZP along its focal distance. The FZP of this work was designed to focus the wavelength between $150 \mathrm{~nm}$ and $450 \mathrm{~nm}$ on the distance of $0.01 \mathrm{~mm}$ to $1 \mathrm{~mm}$ from the lens. Two advantages of embedding a lens on a MEMS actuator include shrinking down the spectrometer's size and eliminating the nonlinearity caused by tilting of the FZP. Since all the corners move along together, and individually controllable, the rotational misalignment is avoided.

Acknowledgments: Authors would like to acknowledge Natural Sciences and Engineering Research Council (NSERC) of Canada and the University of Manitoba Graduate Fellowship (UMGF) for their financial support.

Author Contributions: The MEMS actuator was designed and simulated by Elnaz Afsharipour and the Fresnel zone plate was designed and fabricated by Pawel Glowacki. Cyrus Shafai supervised the research.

Conflicts of Interest: The authors declare no conflict of interest. The founding sponsors had no role in the design of the study; in the collection, analyses, or interpretation of data; in the writing of the manuscript, and in the decision to publish the results.

\section{References}

1. Morrison, J.; Imboden, M.; Little, T.D.; Bishop, D.J. Electrothermally actuated tip-tilt-piston micromirror with integrated varifocal capability. Opt. Express 2015, 23, 9555-9566. doi:10.1364/OE.23.009555.

2. Tormen, M.; Peter, Y.A.; Niedermann, P.; Hoogerwerf, A.; Stanley, R. Deformable MEMS grating for wide tunability and high operating speed. J. Opt. A Pure Appl. Opt. 2006, 8, S337. doi:10.1088/1464-4258/8/7/S07.

3. Xu, Y.; Singh, J.; Jason, T.H.; Ramakrishna, K.; Premchandran, C.S.; Kelvin, C.W.; Kuan, C.T.; Chen, N.; Olivo, M.C.; Sheppard, C.J. MEMS based non-rotatory circumferential scanning optical probe for endoscopic optical coherence tomography. In Proceedings of the SPIE-OSA Biomedical Optics (Optical Society of America), Munich, Germany, 17 June 2007. doi:10.1364/ECBO.2007.6627_33.

4. Magrinyà, J.F. Development of a Compact NDIR Spectrometer Based on MOEMS Components for Fruit Ripening Monitoring. Ph.D. Thesis, Universitat de Barcelona, Barcelona, Spain, 2009.

5. Glowacki, P. An Infrared Spectrometer Based on a MEMS Fresnel Zone Plate for Measuring Dissolved Gases in High Voltage Equipment. Master's Thesis, University of Manitoba, Winnipeg, MB, Canada, 2017. 